\title{
Reflets
}

Revue ontaroise d'intervention sociale et communautaire

\section{Une exploration de modèles pour la coordination des services sociaux aux enfants et adolescents francophones}

\section{Alain Poirier}

Volume 1, numéro 1, printemps 1995

Des pratiques à notre image : défis et ressources

URI : https://id.erudit.org/iderudit/026062ar

DOI : https://doi.org/10.7202/026062ar

Aller au sommaire du numéro

Éditeur(s)

Reflets : Revue ontaroise d'intervention sociale et communautaire

ISSN

1203-4576 (imprimé)

1712-8498 (numérique)

Découvrir la revue

Citer cet article

Poirier, A. (1995). Une exploration de modèles pour la coordination des services sociaux aux enfants et adolescents francophones. Reflets, 1(1), 191-200. https://doi.org/10.7202/026062ar

Tous droits réservés (C) Reflets : Revue ontaroise d'intervention sociale et communautaire, 1995
Ce document est protégé par la loi sur le droit d'auteur. L'utilisation des services d'Érudit (y compris la reproduction) est assujettie à sa politique d'utilisation que vous pouvez consulter en ligne.

https://apropos.erudit.org/fr/usagers/politique-dutilisation/ 


\title{
Une exploration de modèles pour la coordination des services sociaux aux enfants et adolescents francophones
}

\begin{abstract}
Alain Poirier
Consultant, Société d'études et de conseil ACORD

En décembre 1991, un comité d'intervenants francophones, formé à la demande du ministère des Services sociaux et communautaires, retenait les services de notre firme, la Société d'études et de conseil ACORD incorporée, pour réaliser une étude sur les services sociaux en français aux enfants, aux adolescents et aux familles de la région d'Ottawa-Carleton.

L'étude devait inventorier et évaluer les services sociaux présentement offerts en français aux enfants, aux adolescents et aux familles de la région pour ensuite recommander des moyens d'améliorer l'ensemble de ces services, notamment en proposant des modèles de coordination appropriés à la culture et à la langue des clientèles visées.
\end{abstract}

\section{Observations découlant de l'inventaire des services}

La première phase du projet a permis de dresser l'inventaire des services offerts, d'examiner les diverses structures de prestation 
Reflets

et les divers modes d'utilisation des services par les clients francophones.

Cet inventaire portait sur l'activité de 21 agences desservant 12152 clients, dont 4825 étaient inscrits dans des programmes en français. Il est raisonnable de croire que l'activité de ces agences est très représentative des services sociaux aux enfants, aux adolescents et aux familles de la région. En effet, toutes les agences importantes ont répondu à notre questionnaire et bon nombre d'agences plus petites en ont fait autant.

D'après les réponses fournies, les agences bilingues - celles qui offrent des services en français et en anglais - utilisent des approches fort variées dans la prestation des services en français. De ces 14 agences, la moitié disent n'avoir mis en place aucun mécanisme de coordination des services en français. Les autres emploient une variété de moyens: équipes désignées ou programmes parallèles (6), comité sur les services en français (6), coordonnateurs des services en français (4), représentation garantie au conseil d'administration (2). Seulement trois agences ont demandé à être désignées comme offrant des services en français, selon les dispositions de la Loi de 1986 sur les services en français; de celles-ci, une seule a préparé un plan d'implantation des services en français.

Pour ce qui est de la gestion du personnel, encore ici aucune approche commune n'est privilégiée et nous avons remarqué une sous-utilisation des personnes capables de travailler en français. Seulement 231 des 414 employés d'agences bilingues pouvant travailler en français sont affectés, ne serait-ce qu'à temps partiel, à la prestation de services en français.

Finalement, les pratiques élémentaires de bilinguisme institutionnel (publication de documents, affichage dans les bureaux...) ne sont pas choses acquises dans quelques agences.

Les agences (homogènes et bilingues) qui ont répondu à notre questionnaire offrent 177 programmes, dont 77 en français. La gamme des services disponibles en français est cependant plus limitée puisque certains programmes plus spécialisés ne sont disponibles qu'en anglais. De plus, certains programmes offerts 
seulement par les agences bilingues rejoignent proportionnellement moins de francophones que de non-francophones.

Cette situation pourrait s'expliquer, en partie du moins, par la définition des mandats que les agences poursuivent: plusieurs ont tendance à définir leur rôle à partir d'une gamme de services qu'elles souhaitent offrir (mission centered, product driven) plutôt qu'à partir des besoins d'une clientèle spécifique (client centered).

Règle générale, les francophones sont admis et cheminent dans les agences sociales de façon comparable à celle des nonfrancophones. Par contre, on peut observer des différences significatives dans la manière dont les deux groupes linguistiques accèdent aux services. Lorsqu'ils choisissent par eux-mêmes ou qu'ils agissent sur les conseils de leur entourage (parents, amis), les francophones semblent préférer les agences bilingues. Pour leur part, les organismes homogènes francophones, dont les écoles de langue française, préferent de beaucoup référer leurs clients à d'autres agences homogènes, lorsque possible. Les agences bilingues, qui servent plus de francophones et offrent plus de programmes que les agences homogènes, font le contraire, en se référant entre elles les clients francophones.

En travaillant, pour ainsi dire, en circuit fermé, les diverses agences nuisent sans doute au développement de nouveaux services en français en réduisant le poids relatif des francophones dans chacune des agences bilingues.

Finalement, la composition ethno-culturelle de la clientèle francophone servie par les agences change de façon significative et à un rythme plus rapide qu'au sein des communautés nonfrancophones.

\section{Principes devant régir la formulation des modèles}

L'inventaire des services offerts et le profil des clients qui les utilisent nous a permis de formuler un certain nombre de principes 
Reflets

directeurs devant guider la conceptualisation des modèles de réseau de coordination des services.

Nous croyons que le réseau proposé devra favoriser la création de nouveaux services en français et améliorer l'accessibilité des programmes existants, d'abord par une meilleure coordination des ressources en place. Voilà qui pourrait entrainer un redécoupage du mandat des agences et l'établissement d'un nombre accru de partenariats entre celles-ci.

Le nouveau réseau devra encourager les agences à promouvoir leurs services en français, en particulier celles qui offrent des services dans les deux langues. Certaines formes de publicité commune pourront être explorées à cette fin.

Par ailleurs, le réseau devra reconnaître le rôle privilégié que joue le système scolaire comme moyen d'accès aux services sociaux en français. Nonobstant la remarque précédente, d'autres points d'accès devront être mieux utilisés. Le réseau devra favoriser le développement de stratégies de dépistage et d'information communautaire (outreach), notamment auprès de la population préscolaire.

Le réseau proposé devra également être en mesure d'anticiper les besoins d'une clientèle dont le profil ethno-culturel continuera de changer de façon importante au cours des prochaines années et accorder une attention particulière aux besoins des nouveaux Canadiens.

Finalement, et de plus en plus d'exemples dans plusieurs domaines le démontrent, on ne peut assurer une bonne coordination des services en français sans y faire participer les francophones. De même qu'on ne peut ignorer, dans la prestation des services sociaux, les particularités d'une clientèle, on ne peut non plus artificiellement «standardiser» les modes de gestion. Dans un contexte fonctionnel qui reste à définir, le réseau proposé devra confier la gestion des services en français à des francophones.

Ces principes nous ont permis d'ébaucher quatre modèles de réseau de coordination, chacun progressivement plus complet que le précédent. 
Le réseau de coordination intra-agences repose sur l'amélioration du statu quo. Tout en maintenant l'autonomie de mandat des agences existantes, il cherche à mieux utiliser les quelques leviers présentement disponibles (ex.: la désignation d'agences, la standardisation des structures de prestation...) dans le but d'améliorer la prestation des services. Ce modèle pourrait permettre de contrer les lacunes les plus flagrantes du système actuel.

Le réseau de coordination interagences propose des mécanismes plus complets de coordination. Il cherche, par exemple, à systématiser la planification et l'évaluation des services. À long terme, il vise l'amélioration des programmes existants et la création de nouveaux programmes.

Le réseau de coordination supra-agences se compare au précédent à cette différence qu'il s'agit ici d'une coordination plus directe et, au besoin, coercitive. Ce modèle comprendrait donc un quelconque mécanisme d'arbitrage habilité à modifier, en cas de conflit, le mandat des agences afin de rationaliser la prestation des services.

Le réseau de coordination de superagences confie à quelques agences, sans doute homogènes francophones, la responsabilité exclusive ou quasi exclusive de la prestation des services en français. Ce modèle cherche aussi à rationaliser l'activité de ces quelques agences par des contacts périodiques et soutenus.

\section{Consultation sur les modèles}

La deuxième phase de l'étude nous a permis de réaliser une consultation auprès de divers milieux (usagers des services, intervenants actifs dans la prestation de services sociaux en français, porteparole de la communauté francophone) dans le but de juger de la pertinence des modèles ébauchés et, surtout, des principes directeurs les inspirant.

Les usagers et les intervenants consultés ont clairement indiqué qu'il leur importait que les services soient disponibles en français. 
Reflets

Ils ont de plus indiqué qu'ils trouvaient souhaitable que le système accorde une attention particulière aux caractéristiques culturelles des divers groupes ethniques francophones.

L'ensemble de nos interlocuteurs déplore qu'il n'existe pas encore de réseau de services sociaux en français. On croit que les mécanismes de planification sont trop limités et que leur impact est trop peu évident.

Si les dernières années ont été consacrées à la mise en place de services en français, les personnes consultées croient que la priorité des prochaines années doit être l'évaluation et le contrôle de la qualité des services offerts. L'approche qui consiste à offrir des services plutôt qu'à servir une clientèle ne répond plus aux attentes des francophones.

De même, les présents modes de gestion des services ne sont plus acceptables: la gestion des services en français à l'image d'une miniature des services en anglais entraîne une perpétuelle fragilité des services et une constante assimilation des clients francophones dans les services de langue anglaise.

À la lumière des observations ci-haut, la majorité des intervenants consultés concluent à la nécessité de créer des structures homogènes de prestation des services en français. Qu'il s'agisse de créer de nouvelles agences ou des cellules francophones parallèles et largement autonomes au sein d'agences bilingues, de telles structures sont vues comme essentielles à l'évolution des services en français.

Finalement, le statu quo n'est plus acceptable aux personnes consultées. Explicitement ou implicitement, elles rejettent presque à l'unanimité le premier modèle de réseau ébauché plus haut.

\section{Fondements d'un nouveau système}

Les commentaires recueillis au cours de nos consultations nous portent à conclure que le système actuel a été conçu dans le but de favoriser un rattrapage dans la prestation de services sociaux 
en français. Bien qu'il ait largement permis la mise en place de (nouveaux) services de base en français, ce système a depuis atteint sa limite d'efficacité et de croissance.

Le fondement même du système actuel est désuet. L'approche employée en ce moment fractionne la clientèle francophone entre trop d'agences, ralentit l'émergence de ses besoins et la création de nouveaux services, entrave l'accessibilité aux services existants, rend très difficile la coordination d'ensemble et, finalement, engage des dépenses inutiles et des dédoublements coûteux.

Bref, le régime actuel place le pouvoir décisionnel à l'extérieur du cercle des principaux intéressés: les usagers, les intervenants et les professionnels francophones.

En contrepartie, un nouveau réseau de coordination chercherait à regrouper la clientèle francophone et à identifier les moyens permettant de la servir efficacement, malgré son faible poids numérique. Ce réseau permettrait la constitution d'un véritable continuum de services en français et mettrait en valeur la spécificité culturelle et la diversité ethnique de la communauté franco-ontarienne. Finalement, il encouragerait les francophones à prendre en charge le développement de «leurs» services sociaux (responsabilisation, imputabilité).

\section{Élaboration et comparaison des modèles}

La troisième phase de l'étude a servi de synthèse à l'ensemble des travaux menés jusqu'ici. En tenant compte de l'inventaire des services offerts et du profil de la clientèle (phase 1) ainsi que des commentaires recueillis lors des consultations (phase 2), nous avons entrepris de définir plus à fond les modèles, de façon à faire ressortir les avantages et les contraintes de chacun.

Pour ce faire, nous avons d'abord élaboré des critères de comparaison, inspirés des résultats des deux premières phases de l'étude. Ces critères résument les grandes attentes auxquelles un réseau de coordination devrait répondre: faciliter l'accès aux services 
Reflets

existants, faciliter la création de nouveaux services et créer un continuum de services.

Le quatrième et dernier critère nous amenait à évaluer la recevabilité (politique) de chacun des trois modèles de coordination du point de vue des diverses instances concernées par la question. Il nous permettait ainsi d'établir une première mesure de la faisabilité des modèles.

Voici, succinctement, les avantages et les contraintes des divers modèles de réseau de coordination.

Le modèle de réseau de coordination interagences permettrait un meilleur dialogue entre les composantes du réseau et favoriserait certains types de collaborations entre les agences. Son potentiel à coordonner les services serait cependant limité, puisque les fondements du système actuel et les mandats des agences en place demeureraient inchangés. Ce modèle maintiendrait, à toutes fins pratiques, le statu quo en ce qui a trait à la participation des francophones aux divers processus décisionnels.

Le deuxième modèle de réseau de coordination, dit supraagences, permettrait une meilleure coordination des services et une plus grande rationalisation des ressources humaines et financières. Nous croyons que ce modèle pourrait regrouper la population francophone au sein d'un nombre plus restreint d'agences, de façon à favoriser la création de services spécialisés. Ce modèle favoriserait également l'offre active des services disponibles, en particulier auprès des clients plus démunis, ce qui pourrait contribuer à des interventions plus précoces et moins coûteuses. Le modèle supra-agences exigerait que les agences bilingues modifient leurs structures de gestion et acceptent de déléguer certains pouvoirs décisionnels aux gestionnaires francophones.

Le modèle de réseau de coordination superagences permettrait, mieux que tout autre, la planification et la coordination des services en français, ne serait-ce qu'en limitant le nombre d'intervenants engagés dans les processus décisionnels. Ce réseau d'agences pourrait être à l'écoute des besoins des francophones et ainsi faciliter la mise en place de nouveaux services dans le but d'en arriver à un continuum de services attentif aux caractéristiques 
changeantes de la communauté. Il répondrait bien au désir des francophones d'être responsables de la gestion des services qui leur sont offerts. Ce troisième modèle n'améliorerait pas nécessairement l'accessibilité aux services qui seraient concentrés sous la responsabilité de quelques agences. Quant à sa recevabilité, ce modèle est le plus susceptible de susciter la controverse.

\section{Suites à l'étude}

Pour compléter notre travail, nous avons présenté au comité d'intervenants quelques recommandations concernant les suites à donner à l'étude.

Suite aux consultations effectuées et à la comparaison des modèles, il ne fait pour nous aucun doute que le modèle le plus avantageux - dans les circonstances - est celui de réseau de coordination supra-agences.

À notre avis, ce modèle, plus que tout autre, permet de corriger les principales failles du régime actuel et permet de développer une philosophie d'intervention et des approches propres aux francophones. Il favorise la création d'un véritable continuum de services en français qui repose, entre autres, sur une plus grande participation des francophones à la planification et à la gestion des services qui leur sont destinés.

Il est possible que le réseau supra-agences entraîne une redéfinition du mandat des agences offrant des services en français et une redistribution éventuelle des pouvoirs respectifs des diverses composantes du système. Pour ces raisons, il risque de soulever des hésitations et même des objections. Néanmoins, il constitue le plus acceptable des compromis, autant pour les intervenants francophones (dont plusieurs favorisaient plutôt le réseau de superagences) que pour les agences bilingues (qui privilégieraient souvent le statu quo).

Pour faciliter le travail de mise en place du réseau de coordina- 
Reflets

tion, nous avons également présenté une série de recommandations et de suggestions concernant le mandat et les objectifs à court, à moyen et à long termes de ce réseau.

Nous croyons de plus que l'implantation d'un réseau de services aux enfants pourrait servir de première étape à la création d'un véritable réseau social en français, intégrant non seulement les services sociaux aux personnes avec des handicaps, aux adultes et aux personnes du troisième âge, mais aussi les services de santé mentale, de santé, de prévention, d'éducation, de loisirs, etc.

Toutes ces suggestions supposent que le ministère des Services sociaux communautaires s'engage à considérer à leur pleine valeur les recommandations que lui adressera le réseau de coordination. En dernière analyse ce sera toujours le Ministère qui exercera le dernier droit de regard par l'attribution des deniers publics dont il est responsable. 